\title{
Infants Uniquely Express High Levels of RBM3 and Other Cold-Adaptive Neuroprotectant Proteins in the Human Brain
}

\author{
Travis C. Jackson ${ }^{a, b}$ Shawn E. Kotermanski ${ }^{c}$ Patrick M. Kochanek ${ }^{a, b}$ \\ a Safar Center for Resuscitation Research, University of Pittsburgh School of Medicine, UPMC Children's Hospital of \\ Pittsburgh, John G. Rangos Research Center, Pittsburgh, PA, USA; ${ }^{b}$ Department of Critical Care Medicine, University \\ of Pittsburgh, School of Medicine, Scaife Hall, Pittsburgh, PA, USA; ${ }^{C}$ Department of Pharmacology and Chemical \\ Biology, University of Pittsburgh, School of Medicine, Bridgeside Point Building 1, Pittsburgh, PA, USA
}

\section{Keywords}

CIRBP · RTN3 · $\beta$-Klotho · FGF21 - Brain · BA10 •

Hippocampus · Aging

\begin{abstract}
Neuroprotective cold-shock proteins (CSPs) are abundant in the normothermic neonatal rodent brain but decrease with advancing neurodevelopmental age and are low or absent in the adult brain. It has not been established if neurodevelopmental age alters the baseline expression of CSPs in the human brain. Here, we tested the hypothesis that protein levels of RNA-binding motif 3 (RBM3), reticulon-3 (RTN3), and cold-induced RNA-binding protein (CIRBP) are abundant in the normothermic developing human brain but lowto-absent in adults. We also tested if $\beta$-klotho (KLB) is expressed in the developing brain; KLB functions as a coreceptor that controls tissue-specific binding and activity of the systemically circulating thermogenic hormone fibroblast growth factor 21 (FGF21), and is predominantly expressed in the liver, pancreas, and in adipose cells. Methods: Hippocampi and anterior prefrontal cortices (aPFCs/BA10) from a total of 20 male and 20 female subjects were obtained from the NIH NeuroBioBank. CSP and KLB levels were measured
\end{abstract}

\begin{tabular}{ll}
\hline KARGER & $\begin{array}{l}\text { (c) } 2018 \text { The Author(s) } \\
\text { Published by S. Karger AG, Basel }\end{array}$ \\
E-Mail karger@karger.com & $\begin{array}{l}\text { This article is licensed under the Creative Commons Attribution- } \\
\text { NonCommercial-NoDerivatives 4.0 International License (CC BY- } \\
\text { www.karger.com/dne }\end{array}$ \\
& $\begin{array}{l}\text { NC-ND) (http://www.karger.com/Services/OpenAccessLicense). } \\
\text { Usage and distribution for commercial purposes as well as any dis- } \\
\text { tribution of modified material requires written permission. }\end{array}$
\end{tabular}

in: infants $<1$ year old $(n=8)$, toddlers aged $1-2$ years $(n=8)$, children aged $3-5$ years $(n=7), 18$-year-old adolescents $(n=$ $8)$, and adults aged 31-34 years $(n=8)$. An equal number of male and female ( $n=4$ each) samples were pooled into each age group, except in the 3- to 5-year-olds which comprised 3 male and 4 female specimens due to sample availability. In total, 78 whole-brain tissues were dissociated using a beadbased Precellys homogenizer to generate equivalent homogenates, and levels of protein targets subsequently analyzed by Western blotting. Results: Infants had the highest levels of RBM3 and other CSPs in the brain compared to all other ages. In the hippocampus, CSPs were detected predominantly in infants. In the aPFC, CSP levels were highest in infants, moderate-to-low in toddlers/children, and below assay detection limits in adolescents/adults. Germane to the thermogenic FGF21/KLB signaling axis, our results confirm that KLB is absent in the adult hippocampus/aPFC as reported by others. In contrast, we report for the first time that KLB is abundant in the early developing human brain; KLB levels were highest in the infant hippocampus/aPFC and moderately expressed in toddlers. RBM3 is a potent neuroprotective CSP. Thus, the impact of these findings on the observed efficacy of therapeutic hypothermia in neonatal brain injury merits further investigation.

(c) 2018 The Author(s)

Published by S. Karger AG, Basel

Travis C. Jackson, $\mathrm{PhD}$

Safar Center for Resuscitation Research, University of Pittsburgh School of Medicine UPMC Children's Hospital of Pittsburgh, John G. Rangos Research Center - 6th Floor 4401 Penn Avenue, Pittsburgh, PA 15224 (USA)

E-Mail jacksontc@upmc.edu 


\section{Introduction}

Therapeutic hypothermia (TH) is a life-saving intervention that is standard of care for the treatment of neurological damage in term newborns with hypoxic ischemic encephalopathy and also in comatose adults with an out-of-hospital cardiac arrest [1-3]. Clinical trials suggest that the neurological benefits of total body cooling after hypoxia-ischemia is greater for newborns than adults but the molecular underpinnings to explain age-dependent discrepancies in the efficacy of TH in humans have yet to be fully elucidated $[1,3-5]$.

Cold adaptation signaling pathways in mammals are necessarily activated by $\mathrm{TH}$. They involve the recruitment of thermogenic mechanisms such as shivering [6], and the upregulation of cellular cold-shock proteins (CSPs) [7]. Age-dependent differences in the availability of cold stress pathways in the brain may have important implications for the mechanisms of benefit induced by TH in the setting of neonatal versus adult injury of the central nervous system (CNS) [8-10].

A limited number of mammalian CSPs have been identified to date [11-13]. Chief among them is RNAbinding motif 3 (RBM3). RBM3 is a potent neuroprotective CSP, and there is enormous clinical interest in boosting its expression with or without cooling [14]. In rodents, developmental brain age strongly affects baseline RBM3 levels. Normothermic neonatal mice/rats have high levels of RBM3 in the brain whereas levels are lowto-absent in adults $[10,15]$. In addition, the magnitude and duration of RBM3 induction in the retina after an environmental cold stress is greater in newborn rats than adults [16]. Furthermore, we reported that RBM3 protein is robustly increased by cooling to $33^{\circ} \mathrm{C}$ for $48 \mathrm{~h}$ in immature day in vitro (DIV)6 rat cortical neurons but not in mature DIV26 neurons [17]. Together, the data show

Fig. 1. Developmental time course of RNA-binding motif 3 (RBM3) expression in the human hippocampus. a Western blots show that a polyclonal anti-RBM3 antibody and PVDF membrane previously validated by our group (in rodents) also detects human RBM3 (approx. $17 \mathrm{kDa}$ ) in human neuronal SHSY5Y cells given 48-h hypothermia. Two different RBM3-targeting shRNAs were tested to confirm target specificity. Lentivirus/shRNA-B blocked RBM3 upregulation. The asterisk indicates a higher-molecularweight (approx. 18-19 kDa) RBM3 protein which was also increased by cooling and decreased by shRNA knockdown. b Table of subject/specimen characteristics. c Representative Western blots show hippocampal RBM3 levels in infants, toddlers, children, that developmental brain age is a major factor in the study of CSP-based therapies in rodents. However, it has not been elucidated if developmental age is equally important in humans germane to the study of brain CSPs.

Here, we tested the hypothesis that RBM3, reticulon-3 (RTN3), and cold-induced RNA-binding protein (CIRBP) levels are high in the normothermic infant human brain but low-to-absent in adults. Moreover, with the exception of shivering mechanisms [6], little work has been done to investigate the presence of thermogenesisassociated intracellular signaling pathways in the brain which may be affected by total body cooling. Because infants uniquely rely on brown adipose tissue (BAT) to maintain body temperature $[8,9]$, and because FGF21 (a BAT-stimulating hormone) increased RBM3 levels in immature rat cortical neurons in vitro [17], we also tested the hypothesis that $\beta$-klotho (KLB) which exclusively governs FGF21 activity in vivo $[18,19]$, is detectable in the infant brain.

\section{Materials and Methods}

\section{Chemicals and Reagents}

PVDF Membranes

To detect RBM3, we used 0.45- $\mu \mathrm{M}$-pore-size Hybond-P, Cat\# RPN2020F, Lot\# NH1288 (GE Healthcare; Pittsburgh, PA, USA). To detect RTN3, CIRBP, and KLB, we used 0.2- $\mu \mathrm{M}$-pore-size mdi membrane, Cat\# SVFX8301XXXX101, Lot\# VA760606L (mdi Membrane Technologies; Harrisburgh, PA, USA).

\section{Antibodies}

We made use of anti-RBM3, Cat\# 14363-1-AP, Lot\# N/A (Proteintech; Chicago, IL, USA); anti-RTN3, Cat\# OABB01421, Lot\# 0221412 c015615 (AVIVA Systems Biology; San Diego, CA, USA); anti-CIRBP clone EPR18783, Cat\# ab191885, Lot\# GR239199-2 (Abcam; Cambridge, MA, USA); anti-KLB, Cat\# ab106794, Lot\# GR3200089-1 (Abcam); anti-a-tubulin, Cat\# 2144, Lot\# 5 (Cell Signaling Technology; Danvers, MA, USA). adolescents, and adult subjects ( $n=4$ /group). The corresponding NIH NBB deidentified subject ID is indicated above each sample. d Representative total protein stain confirms equal protein loading and membrane transfer across groups. e The graph shows that RBM3 levels are highest in the infant hippocampus ( $n=8$ /group) compared to toddlers ( $n=8 /$ group), children ( $n=7 /$ group), adolescents ( $n=8$ /group), and adults ( $n=8 /$ group). Normalized densitometry values were analyzed by Kruskal-Wallis one-way ANOVA. * significance (Dunn's post hoc multiple-comparisons test). Data were significant at $p<0.05$. Graphs show mean (green) + SEM (red).

(For legend see next page.) 
Human Tissues

NIH NeuroBioBank Tissues

Experiments with human tissues were approved by the University of Pittsburgh Committee for Oversight of Research and Clinical Training Involving Decedents (CORID). An application re- questing 80 brain specimens, 40 hippocampi, and 40 Brodmann area 10, i.e., anterior prefrontal cortices (aPFCs) originating from 40 subjects categorized as unaffected controls, preselected by age and gender, was submitted to the NIH NeuroBioBank (NBB) repository for review. The request was approved with minor modi-

\section{Human Neuronal SHSY5Y Cells}

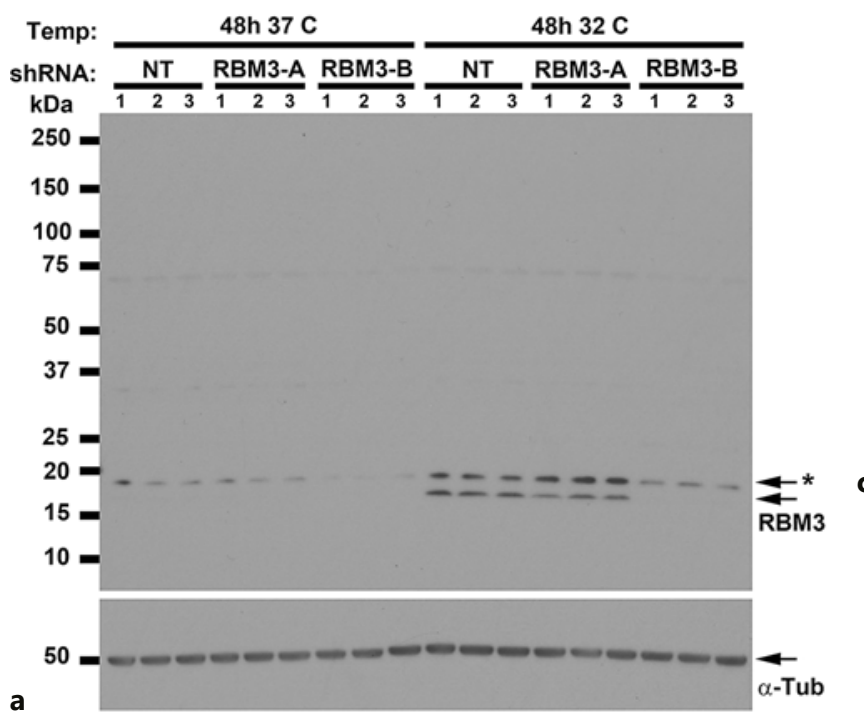

NIH Neurobiobank Sample Characteristics (Unaffected Controls)

\begin{tabular}{|c|c|c|c|c|c|}
\hline & $<1 \mathrm{yr}$ & $1-2 \mathrm{yr}$ & $3-5 \mathrm{yr}$ & $18 \mathrm{yr}$ & $31-34 \mathrm{yr}$ \\
\hline \multicolumn{6}{|l|}{ Race } \\
\hline Caucasian & $5 / 8(63 \%)$ & $5 / 8(63 \%)$ & $5 / 8(63 \%)$ & $4 / 8(50 \%)$ & $6 / 8(75 \%)$ \\
\hline African Amer. & $2 / 8(25 \%)$ & $3 / 8(38 \%)$ & $3 / 8(38 \%)$ & $1 / 8(13 \%)$ & $2 / 8(25 \%)$ \\
\hline Hispanic & $0 / 8(0 \%)$ & $0 / 8(0 \%)$ & $0 / 8(0 \%)$ & $2 / 8(25 \%)$ & $0 / 8(0 \%)$ \\
\hline Indian Sub. & $0 / 8(0 \%)$ & $0 / 8(0 \%)$ & $0 / 8(0 \%)$ & $1 / 8(13 \%)$ & $0 / 8(0 \%)$ \\
\hline Other & $1 / 8(13 \%)$ & $0 / 8(0 \%)$ & $0 / 8(0 \%)$ & $0 / 8(0 \%)$ & $0 / 8(0 \%)$ \\
\hline \multicolumn{6}{|l|}{ Gender } \\
\hline Female & $4 / 8(50 \%)$ & $4 / 8(50 \%)$ & $4 / 8(50 \%)$ & $4 / 8(50 \%)$ & $4 / 8(50 \%)$ \\
\hline Male & $4 / 8(50 \%)$ & $4 / 8(50 \%)$ & $4 / 8(50 \%)$ & $4 / 8(50 \%)$ & $4 / 8(50 \%)$ \\
\hline \multicolumn{6}{|l|}{ Age (yr) } \\
\hline Mean & $0.19 \pm 0.09^{\#}$ & $1.47 \pm 0.45$ & $4.42 \pm 0.93$ & $18.58 \pm 0.29$ & $33.23 \pm 1.40$ \\
\hline \multicolumn{6}{|l|}{ Tissue } \\
\hline Hippocampus & $8 / 8$ & $8 / 8$ & $7 / 8^{*}$ & $8 / 8$ & $8 / 8$ \\
\hline $\mathrm{BA}-10$ & $8 / 8$ & $8 / 8$ & $7 / 8^{*}$ & $8 / 8$ & $8 / 8$ \\
\hline \multicolumn{6}{|l|}{ PMI (hr) } \\
\hline Mean & 24 & 24.8 & 19.9 & 22.8 & 22.0 \\
\hline Median & 28.5 & 24.0 & 19.5 & 21.0 & 22.5 \\
\hline
\end{tabular}

* Hippocampus was not availble for one patient in the $3-5 \mathrm{yr}$ group. BA-10 was not available for one patient in the 3-5 yr group.

b \# Exact age missing for one infant

\section{Human Hippocampal Tissues}

Nonshivering Shivering Thermogenesis
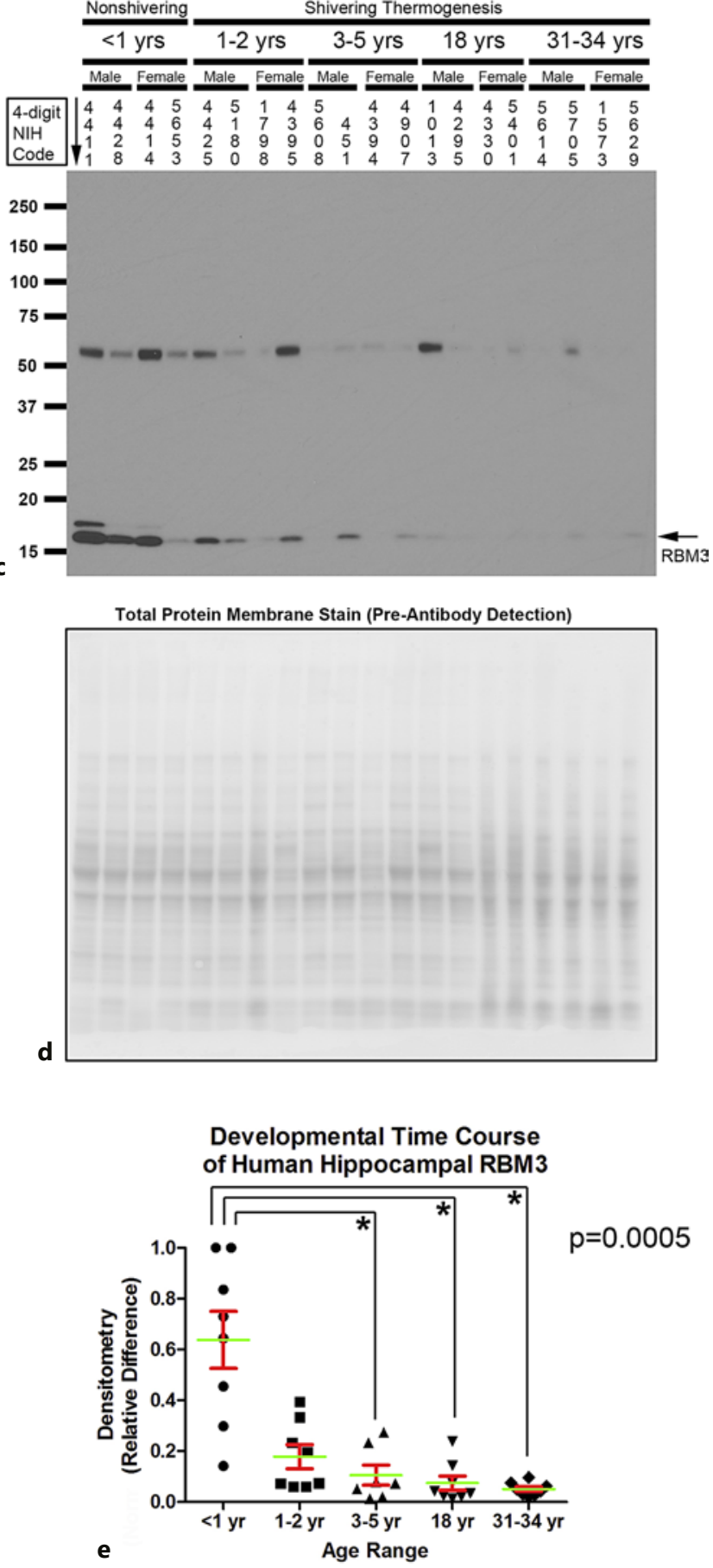
fications based on sample availability. A total of 78 frozen brain specimens (39 hippocampi and 39 aPFCs) from 40 patients were shipped to the University of Pittsburgh and stored at $-80^{\circ} \mathrm{C}$. Hippocampal tissues were representative of the whole anatomical area and were not enriched for a particular subregion. The full list of

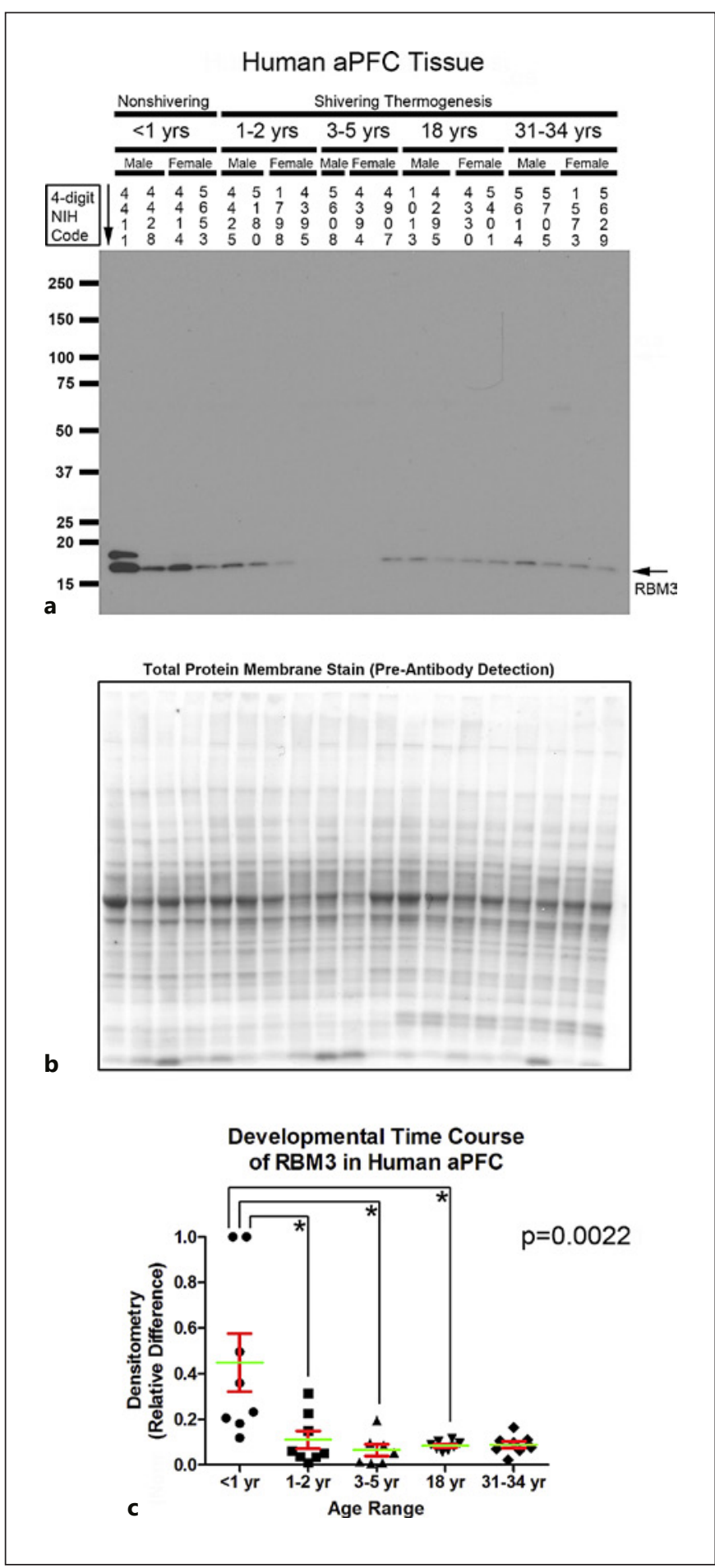

deidentified NBB identification numbers (https://neurobiobank. nih.gov) associated with specimens used in this study is available from the corresponding author upon request. In the group of 3- to 5 -year-olds, 1 male subject (ID 4332) did not have a hippocampus available for analysis, and another male subject (ID 451) did not have an aPFC available for analysis. Frozen tissues were thawed on ice in Precellys 24 homogenizing CK14-2-mL tubes (Bertin Instruments; Rockville, MD, USA), containing $1 \mathrm{~mL}$ (hippocampal specimens) or $700 \mu \mathrm{L}$ (aPFC specimens) ice-cold radio immunoprecipitation assay (RIPA) buffer, and supplemented with $1 \times$ EDTA, $2 \times$ protease inhibitors, and $2 \times$ phosphatase inhibitors (PIERCE, Rockford, IL, USA). Tubes were quickly transferred (20 at a time) into a Precellys 24 homogenizer (Bertin Instruments), established under a laminar flow hood, and mechanically lysed for $10 \mathrm{~s}$ at 5,500 rpm. Samples were immediately returned to ice for $5 \mathrm{~min}$, lysed again for $10 \mathrm{~s}$ at 5,500 rpm, again returned to ice, and homogenates transferred into new $1.5-\mathrm{mL}$ conical tubes. Homogenates were vortexed and returned to a $-80{ }^{\circ} \mathrm{C}$ ultralow overnight for additional cryolysis. Homogenates were thawed on ice, vortexed for 10 min, centrifuged $15 \mathrm{~min}$ at $16,000 \mathrm{rcf}$ and $4{ }^{\circ} \mathrm{C}$, and the supernatants transferred into new $1.5-\mathrm{mL}$ tubes. Protein concentrations of stock homogenates were measured by BCA assay (PIERCE). Protein concentrations were adjusted to approximately $3.5 \mu \mathrm{g} / \mu \mathrm{L}$ by dilution in additional RIPA buffer (supplemented with $1 \times$ EDTA, $2 \times$ protease inhibitors, and $2 \times$ phosphatase inhibitors). Adjusted homogenates were reanalyzed by BCA to ensure accurate protein loading and stored at $-80^{\circ} \mathrm{C}$ until use.

\section{Lentivirus Production}

BSL2 work was approved by the Institution Biosafety Committee of the University of Pittsburgh. Human-specific RBM3 shRNA knockdown expression plasmids (TL309915-A and TL309915-B) were purchased from Origene (Rockville, MD, USA). Packaging plasmids were also purchased from Origene (Cat\# TR30037). A detailed description of our lentivirus production procedures has been reported elsewhere [20]. In brief, 2 different RBM3 shRNA expression plasmids (A and B) and a nontargeting (NT) shRNA control plasmid (Cat\# TR30021) were maxiprepped to obtain high-quality DNA. Expression and packaging plasmids were mixed with MegaTran 1.0 transfection reagent and incubated with HEK293ta cells (Genecopoeia; Rockville, MD, USA) for approximately $12 \mathrm{~h}$. Transfection medium was removed and re-

Fig. 2. Developmental time course of RNA-binding motif 3 (RBM3) expression in the human anterior prefrontal cortex (aPFC). a Representative Western blots show aPFC RBM3 levels in infants, toddlers, children, adolescents, and adult subjects $(n=$ 3 /group in children, $n=4$ /group in all other age groups). The corresponding NIH NBB deidentified subject ID is indicated above each sample. b Representative total protein stain confirms equal protein loading and membrane transfer across groups. c The graph shows that RBM3 levels are highest in the infant aPFC $(n=$ 8 /group) compared to toddlers ( $n=8$ /group), children $(n=7 /$ group), adolescents ( $n=8$ /group), and adults ( $n=8$ /group). Normalized densitometry values were analyzed by Kruskal-Wallis one-way ANOVA. * significance (Dunn's post hoc multiple-comparisons test). Data were significant at $p<0.05$. Graphs show mean (green) + SEM (red). 
placed with fresh Opti-MEM, 10\%FBS, and penicillin-streptomycin. Viral soup was harvested at around 36 and $48 \mathrm{~h}$ after transfection. For each vector, medium collections were combined, filtered, and ultracentrifuged to obtain the viral pellet. The pellet was resuspended in $200 \mu \mathrm{L}$ Opti-MEM medium, aliquoted, and stored

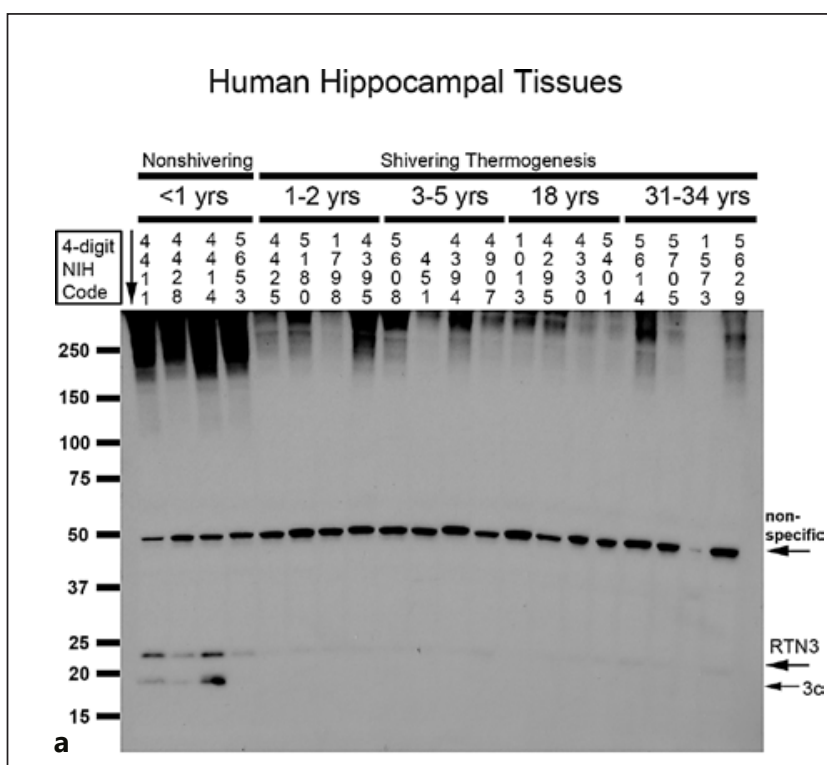

Total Protein Membrane Stain (Pre-Antibody Detection)

b
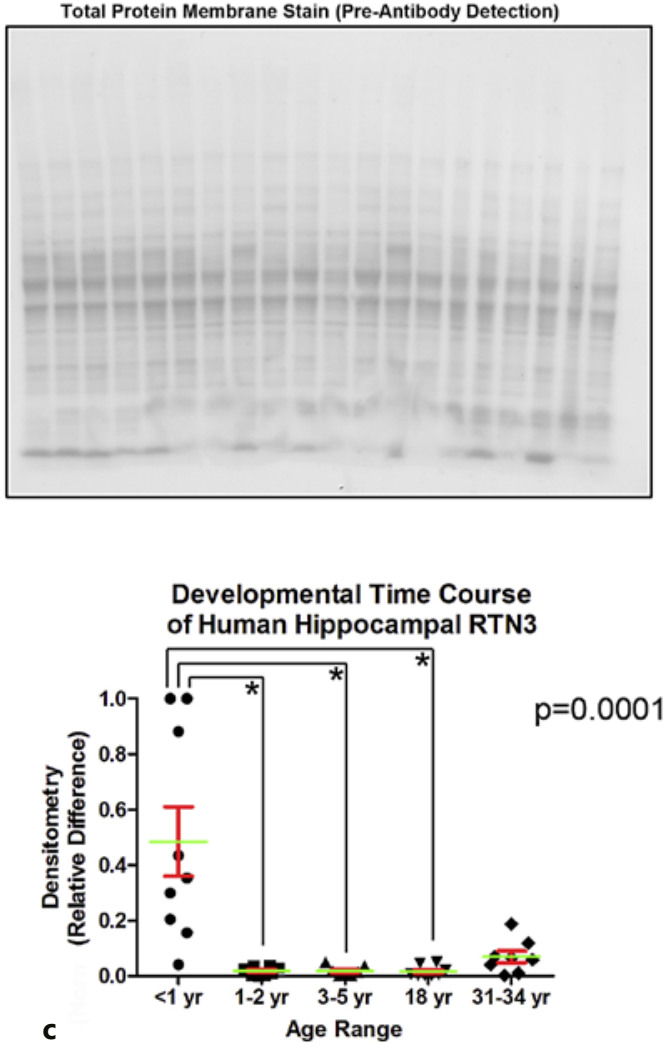

at $-80^{\circ} \mathrm{C}$. Viral titer was estimated by ELISA as reported previously [20].

\section{Cell Culture and Hypothermia Studies}

Wild-type HEK293ta cells (for virus production) and human neuronal SHSY5Y cells (for virus transduction) (ATCC; Manassas, VA, USA) were propagated in Opti-MEM, 10\% FBS, and penicillin-streptomycin. Confluent cells were dissociated by trypsinization. SHSY5Y cell concentrations were measured on a Cellometer (Nexcelom Bioscience; Lawrence, MA, USA). $5 \times 10^{6} /$ cells were mixed with 60 multiplicity of infection (MOI) lentivirus. Transduced cells were seeded onto new T75 flasks and proliferated over $72 \mathrm{~h}$. The medium was subsequently replaced $3 \times$ at 24 -h intervals, starting immediately after the initial 72 -h transduction period, and contained $1 \mu \mathrm{g} / \mathrm{mL}$ puromycin to eliminate nontransduced cells. Following puromycin selection, cells were trypsinized and subsequently seeded in fresh medium (without puromycin) onto polyD-lysine-coated 6-well plates $\left(2 \times 10^{6} /\right.$ well $)$. Hypothermia experiments were initiated $24 \mathrm{~h}$ later. For each vector condition (NT shRNA, RBM3 shRNA-A, or RBM3 shRNA-B), 1 set of 6-well plates was incubated for $48 \mathrm{~h}$ at $37^{\circ} \mathrm{C}$, and another set at $32^{\circ} \mathrm{C}$. Following temperature treatments, cells were washed with ice-cold PBS, immediately harvested in RIPA buffer (supplemented with EDTA, protease inhibitors, and phosphatase inhibitors), and stored at $-80^{\circ} \mathrm{C}$. Samples were homogenized by sonication (approx. $20 \mathrm{~s}$ ), centrifuged for $10 \mathrm{~min}$ at $16,000 \mathrm{rcf}$ and $4^{\circ} \mathrm{C}$, and protein concentrations in supernatants analyzed by BCA assay.

\section{Western Blot}

Protein analysis was done as previously described by our group [17]. In brief, samples $(20 \mu \mathrm{g} /$ well $)$ were mixed with an equal volume of $2 \times$ Laemmli sample buffer (supplemented with 2-ME) and boiled $5 \mathrm{~min}$ at $95^{\circ} \mathrm{C}$. Protein standards/samples were loaded onto 26-well precast TGX gels (BioRad, Hercules, CA, USA) and run at $200 \mathrm{~V}$ in a Criterion ${ }^{\mathrm{TM}}$ Cell (BioRad). Protein was transferred to PVDF membranes in a plate-electrode Criterion ${ }^{\mathrm{TM}}$ Blotter (Bio$\mathrm{Rad}$ ), and run for $40 \mathrm{~min}$ at $100 \mathrm{~V}$ and $4{ }^{\circ} \mathrm{C}$. Membranes were stained with reversible swift membrane stain (Fisher Scientific), images collected on a 600-dpi flat-bed scanner, destained, and blocked for $1 \mathrm{~h}$ at RT in $19 \mathrm{~mL}$ of $7.5 \%$ milk dissolved in Tween-20/ Tris-buffered saline (T-TBS). We and others showed that total protein stain is a more accurate method to standardize protein loading versus traditional housekeeping genes [21-25]. House-

Fig. 3. Developmental time course of reticulon-3 (RTN3) expression in the human hippocampus. a Representative Western blots show hippocampal RTN3 levels in infants, toddlers, children, adolescents, and adult subjects ( $n=4$ /group). The corresponding NIH NBB deidentified subject ID is indicated above each sample. b Representative total protein stain confirms equal protein loading and membrane transfer across groups. $c$ The graph shows that RTN3 levels are highest in the infant hippocampus ( $n=8$ /group) compared to toddlers ( $n=8 /$ group), children ( $n=7 /$ group), adolescents ( $n=8$ /group), and adults ( $n=8$ /group). Normalized densitometry values were analyzed by Kruskal-Wallis one-way ANOVA. * significance (Dunn's post hoc multiple-comparisons test). Data were significant at $p<0.05$. Graphs show mean (green) + SEM (red). 
keeping genes are also affected by developmental age in the brain [26]. Primary antibodies (in $15 \mathrm{~mL}$ milk and T-TBS) were incubated overnight on a rocker at $4{ }^{\circ} \mathrm{C}$. Membranes were washed $3 \times$ (in $50 \mathrm{~mL}$ TBS), incubated $2 \mathrm{~h}$ with secondary antibodies $(1: 15,000$ in milk and T-TBS), washed $3 \times$ more in TBS, and incubated ap-

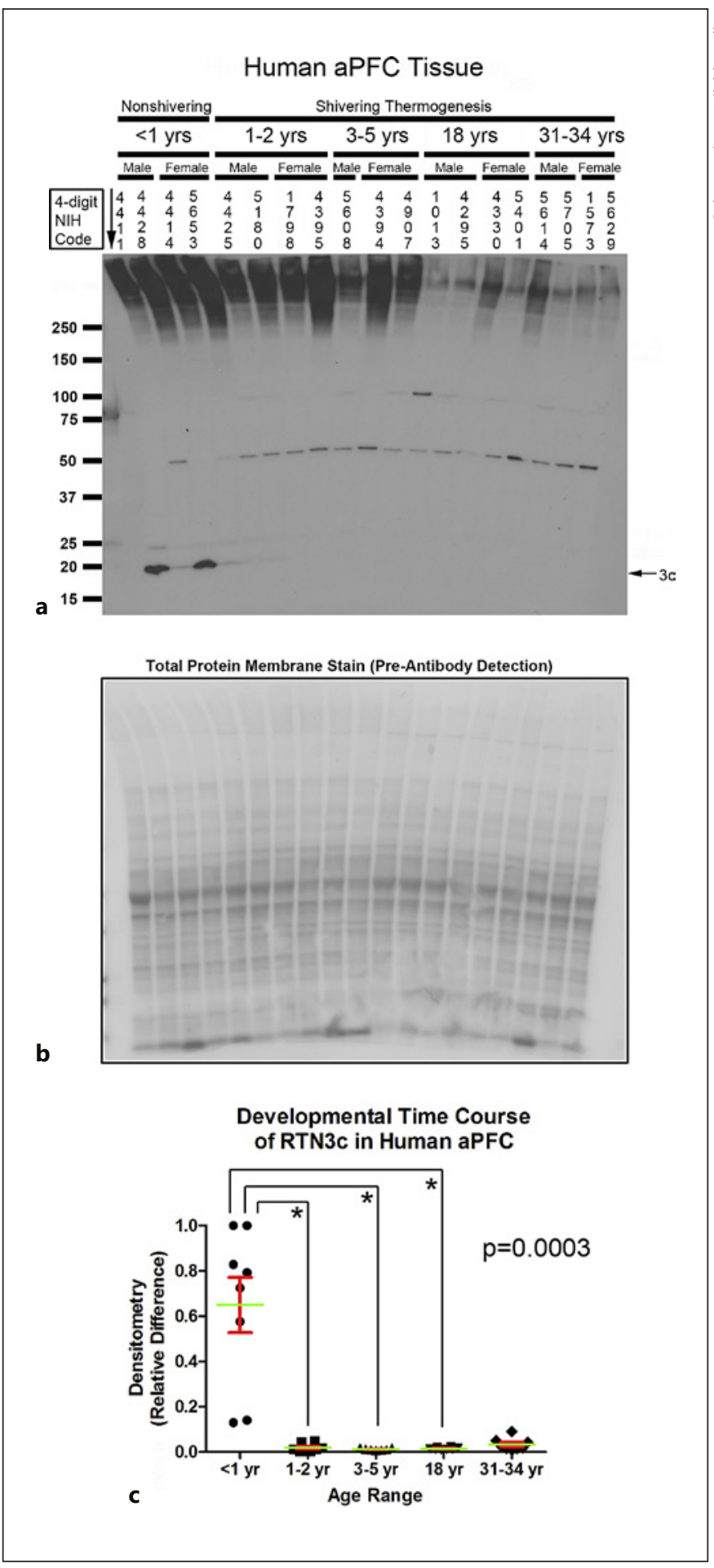

proximately 2 min in ECL-2 Western blotting substrate (Fisher Scientific). Films were exposed in a dark room, collected on a 600pdi flat-bed scanner, and compiled in Photoshop (Adobe; San José, CA, USA). All figures show original unaltered images (i.e., blots and total protein stains were not contrast/brightness modified. A total of 8 independent Western blot experiments were done. Per experiment, 39 samples from either the hippocampus or aPFC were divided equally (by age and gender) across $2 \times 26$-well Criterion gels. A single primary protein target was analyzed per Western blot experiment. Densitometry of proteins and total stain were obtained using UN-SCAN-IT software (Silk Scientific; Orem, UT, USA). To correct for loading/transfer errors (i.e., to standardize), target protein densitometry was divided by the densitometry of the total protein stain corresponding to each lane within the respective membrane. Standardized densitometic values for each blot were normalized by dividing by the largest intra-blot value (i.e., the highest value in each blot was set to $100 \%$ ). Data are expressed as the relative difference in target expression across samples. Normalized values (across the 39 samples) were pooled for statistical analysis.

\section{Statistics}

Normalized densitometry values expressed as the relative difference in target levels were analyzed by nonparametric tests. The effect of developmental age on target expression was analyzed using the Kruskal-Wallis 1-way-ANOVA on ranks test followed by Dunn's post hoc multiple-comparisons test. Gender was analyzed by the Mann-Whitney U test. Data were analyzed using GraphPad Prism software (GraphPad Inc., La Jolla, CA, USA). Data are significant at $\mathrm{p}<0.05$. Graphs show mean and SEM.

\section{Results}

Analysis of RBM3 in Human Hippocampus and aPFC Stable human RBM3 knockdown cells were generated to validate the reagents used to detect cold-induced human RBM3. As expected, an approximately $17-\mathrm{kDa}$ band robustly increased in NT-vector control neurons subjected to $48 \mathrm{~h}$ of hypothermia but was not detected in normo-

Fig. 4. Developmental time course of reticulon-3 (RTN3) expression in the human anterior prefrontal cortex (aPFC). a Representative Western blots show aPFC RTN3 levels in infants, toddlers, children, adolescents, and adult subjects ( $n=3$ /group in children, $n=4$ /group in all other ages). The corresponding NIH NBB deidentified subject ID is indicated above each sample. b Representative total protein stain confirms equal protein loading and membrane transfer across groups. $\mathbf{c}$ The graph shows that RTN3 levels are highest in the infant aPFC ( $n=8 /$ group) compared to toddlers ( $n=8$ /group), children ( $n=7 /$ group), adolescents ( $n=8$ /group), and adults ( $n=8$ /group). Normalized densitometry values were analyzed by Kruskal-Wallis one-way ANOVA. * significance (Dunn's post hoc multiple-comparisons test). Data were significant at $p<0.05$. Graphs show mean (green) + SEM (red). 
thermic neurons or cooled cells transduced with the RBM3-targeting shRNA-B (Fig. 1a). RBM3-targeting vector shRNA-A was ineffective and did not block increased RBM3 levels after cooling.

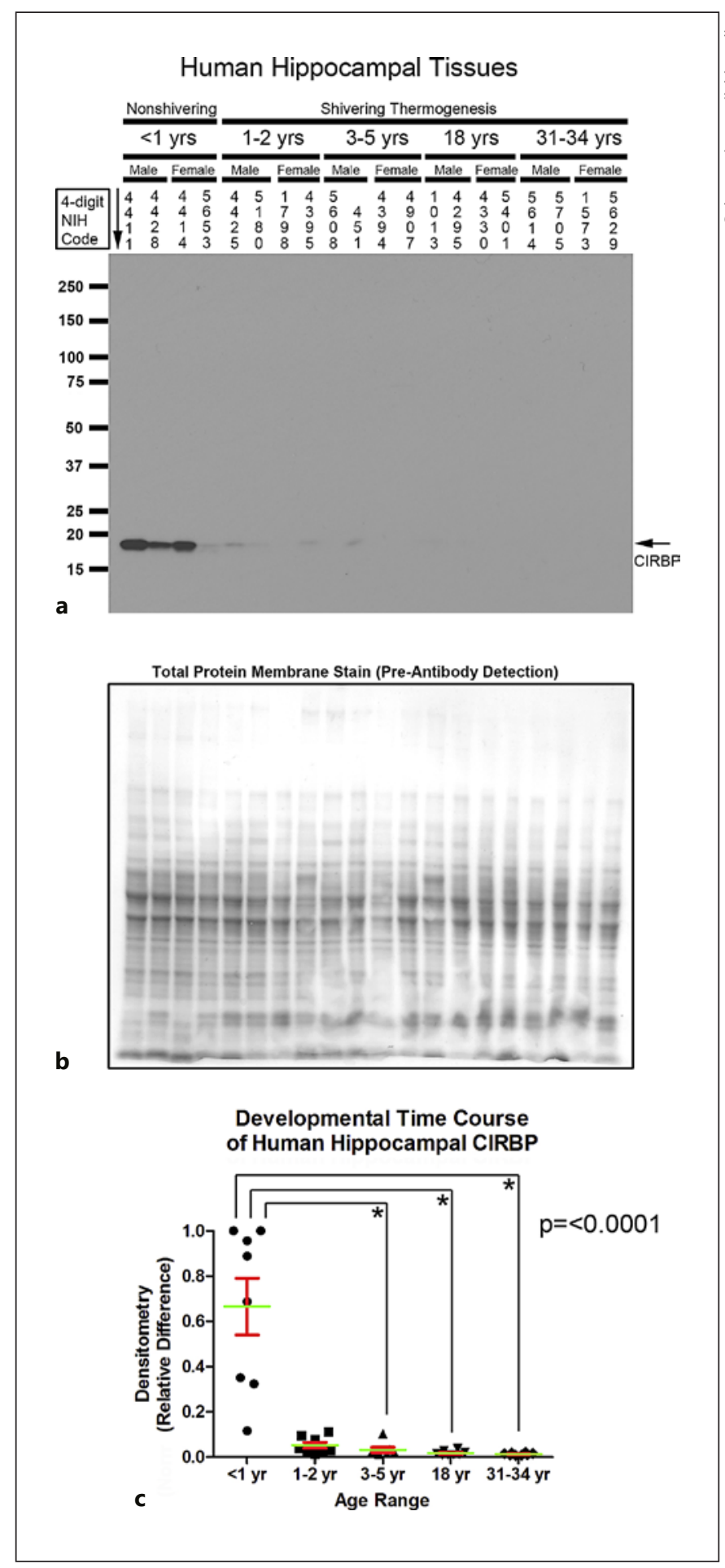

RBM3 Levels in the Human Brain
Seventy-eight high-quality frozen brain tissue specimens (39 hippocampi and 39 aPFCs) from 40 subjects were acquired from the NIH NBB $(n=7-8$ per age group). Figure $1 \mathrm{~b}$ shows subject/specimen characteristics for each group. Hippocampal RBM3 levels (approx. $17 \mathrm{kDa}$ ) were increased in infants compared to all other age groups (Fig. 1c-e). aPFC RBM3 levels were also increased in infants versus toddlers, children, and adolescents (Fig. 2a-c).

\section{Analysis of RTN3 and CIRBP in Human \\ Hippocampus and aPFC}

Hippocampal RTN3 (approx. $23 \mathrm{kDa}$ ) was increased in infants compared to toddlers, children, and adolescents (Fig. 3a-c). RTN3c is the smallest variant. Depending on the tissue, it appears as a single (approx. $19 \mathrm{kDa}$ ) or double (approx. 16/19 kDa) band [27]. The 19-kDa RTN3c protein was also increased in the hippocampus of infants versus toddlers and children $(p=0.0016)$. In contrast, a nonspecific approximately $49-\mathrm{kDa}$ band was decreased in infants versus toddlers, children, and adults ( $p=0.0016)$; the age-dependent increase in the levels of the nonspecific band contrasted with the recurring (opposite) pattern of decreased CSPs with advancing age. In the aPFC, RTN3 (approx. $23 \mathrm{kDa}$ ) was below the level of detection in all samples except subject 4411, precluding statistical analysis (Fig. 4a). RTN3c (approx. $19 \mathrm{kDa}$ ) was increased in the aPFC of infants versus toddlers, children, and adolescents (Fig. 4a-c).

Developmental changes in CIRBP (approx. $17 \mathrm{kDa}$ ) were similar to in RBM3. Hippocampal CIRBP was increased in infants versus children, adolescents, and adults (Fig. 5a-c). In the aPFC, CIRBP was also increased in infants versus children, adolescents, and adults (Fig. 6a-c).

Fig. 5. Developmental time course of cold-induced RNA-binding protein (CIRBP) expression in the human hippocampus. a Representative Western blots show hippocampal CIRBP levels in infants, toddlers, children, adolescents, and adult subjects $(n=4$ / group). The corresponding NIH NBB deidentified subject ID is indicated above each sample. b Representative total protein stain confirms equal protein loading and membrane transfer across groups. c The graph shows that CIRBP levels are highest in the infant hippocampus ( $n=8$ /group) compared to toddlers $(n=8$ / group), children ( $n=7 /$ group), adolescents ( $n=8$ /group), and adults ( $n=8$ /group). Normalized densitometry values were analyzed by Kruskal-Wallis one-way ANOVA. * significance (Dunn's post hoc multiple-comparisons test). Data were significant at $p<$ 0.05. Graphs show mean (green) + SEM (red). 
Analysis of KLB in the Human Hippocampus and aPFC

Hippocampal KLB levels (approx. $120 \mathrm{kDa}$ ) were increased in infants versus children, adolescents, and adults (Fig. $7 \mathrm{a}-\mathrm{c})$. Hippocampal KLB levels were also increased

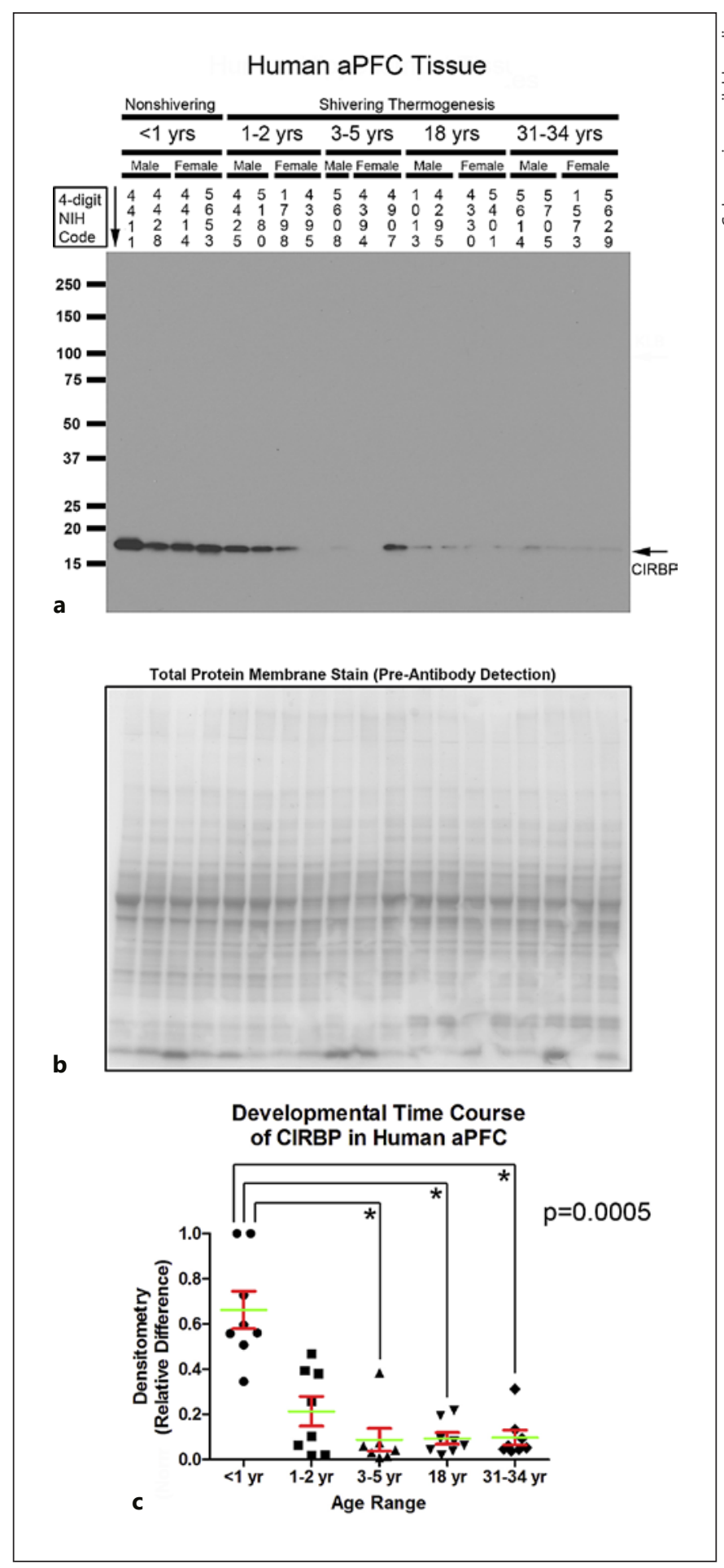

in toddlers compared to adolescents (Fig. $7 \mathrm{a}-\mathrm{c}$ ). In the aPFC, KLB levels were increased in infants versus adolescents and adults (Fig. 8a-c). aPFC KLB levels were also increased in toddlers versus adults (Fig. 8a-c). No significant gender differences (19 males vs. 20 females) were detected for any of the CSP or KLB levels in either the hippocampus or aPFC (hippocampus: $\mathrm{RBM} 3, p=0.5741$; RTN3, $p=0.8661$; CIRBP, $p=0.2216$; KLB, $p=0.4072$; aPFC: RBM3, $p=0.8994$; RTN3, $p=0.9776$; CIRBP, $p=$ $0.5458 ; \mathrm{LB}, p=0.7044)$.

\section{Discussion}

This is the first large-scale investigation of baseline CSP expression in the human brain. The hippocampus was studied because it is a major target of CNS therapies (such as neuroprotective cooling [28]). The aPFC was studied because it does not mature until late adolescence $[29,30]$; we thus hypothesized that developmental effects on CSPs might persist longer in that brain region. We focused our analysis on the mammalian CSPs, RBM3, RTN3, and CIRBP, which have a known function in disease/injury.

\section{Implications of Baseline CSP Expression in the Brain on Therapeutic Cooling in Humans}

RBM3 decreased neuronal damage in acute and chronic models of CNS injury, and there is enormous clinical interest in boosting its expression after a brain injury [14, 15]. RTN3 (approx. $23 \mathrm{kDa}$ ) is the newest addition to the neuroprotective group of CSPs, and it decreased neurological damage in a severe model of neurodegenerative prion disease [12].

Fig. 6. Developmental time course of cold-induced RNA-binding protein (CIRBP) expression in the human anterior prefrontal cortex (aPFC). a Representative Western blots show aPFC CIRBP levels in infants, toddlers, children, adolescents, and adult subjects ( $n=3$ /group in children, $n=4$ /group in all other ages). The corresponding NIH NBB deidentified subject ID is indicated above each sample. b Representative total protein stain confirms equal protein loading and membrane transfer across groups. c The graph shows that CIRBP levels are highest in the infant aPFC ( $n=8 /$ group) compared to toddlers ( $n=8 /$ group), children ( $n=7$ /group), adolescents $(n=8 /$ group $)$, and adults $(\mathrm{n}=8 /$ group). Normalized densitometry values were analyzed by Kruskal-Wallis one-way ANOVA. * significance (Dunn's post hoc multiple-comparisons test). Data were significant at $p<0.05$. Graphs show mean (green) + SEM (red). 
In rodents, neurodevelopmental age strongly affects the baseline expression of neuroprotective CSPs. Both RBM3 and RTN3c (approx. $19 \mathrm{kDa}$ ) levels are abundant in the young developing normotheric rat/mouse brain

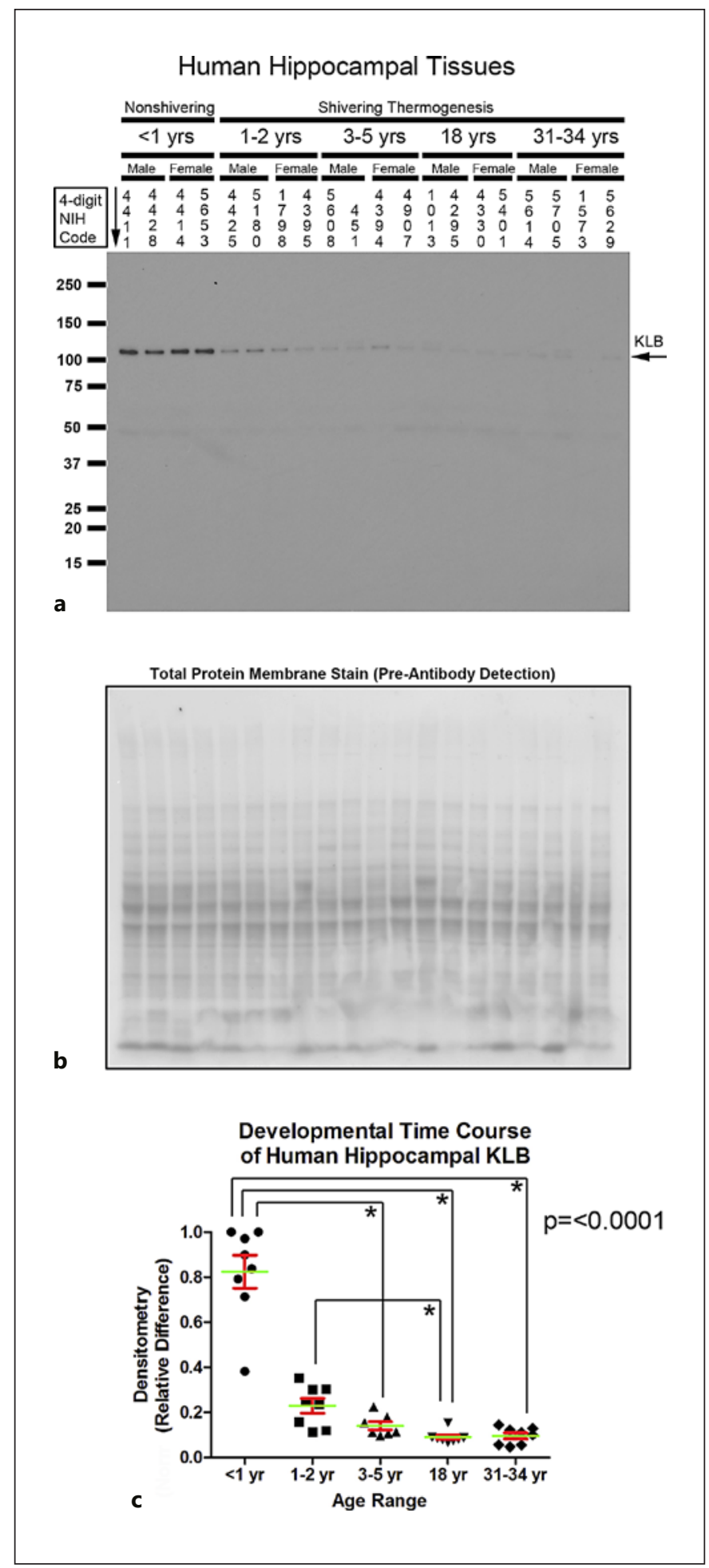

RBM3 Levels in the Human Brain whereas levels are low-to-absent in adults [10, 15, 27]. Our results confirm that developmental expression of RBM3/ RTN3 in humans is similar to observations in rodents. Furthermore, in rodents, the amplified state of baseline CSP expression in the developing brain/neurons is associated with an even greater magnitude of induction after cooling $[16,17]$. Based on these findings, we speculate that humans may have similar CSP physiology, and that total body cooling in newborns may increase neuroprotective CSPs to a greater magnitude versus in adults. More studies are needed to critically evaluate that hypothesis.

Not all CSPs are beneficial in the setting of disease or injury. For instance, CIRBP is deleterious in cancer [31], hemorrhagic shock [32], sepsis [32], and brain ischemia [33]. Interventions that block proinflammatory CIRBP could be beneficial during neuroprotective total body cooling. However, future studies are needed to determine if CIRBP is proinflammatory in the setting of developmental brain injury, and/or has other functions yet to be identified.

\section{Secondary Analysis of KLB}

Thermogenic cell signaling pathways have pleiotropic effects beyond heat production, and may be harmful or beneficial to the injured brain. In human newborns, body temperature is regulated by nonshivering thermogenesis via the activation of BAT $[8,9]$. After 1 year of age, thermoregulatory mechanisms switch to muscle-shivering and a distinct form of nonshivering thermogenesis in the skeletal muscle [34]. FGF21 is an endocrine hormone that activates BAT and raises body temperature in neonatal mice [18]. In addition, in human newborns, endogenous circulating FGF21 levels spike during the first week of life and remain at high concentrations for at least a year [35]. In contrast, in adult mice, FGF21 is dispensable for body temperature maintenance [36].

Fig. 7. Developmental time course of $\beta$-klotho (KLB) expression in the human hippocampus. a Representative Western blots show KLB levels in infants, toddlers, children, adolescents, and adult subjects ( $n=4 /$ group). The corresponding NIH NBB deidentified subject ID is indicated above each sample. b Representative total protein stain confirms equal protein loading and membrane transfer across groups. c The graph shows that KLB levels are highest in the infant hippocampus ( $n=8 /$ group) compared to toddlers $(n=$ 8 /group), children ( $n=7 /$ group), adolescents ( $n=8$ /group), and adults ( $n=8$ /group). Normalized densitometry values were analyzed by Kruskal-Wallis one-way ANOVA. * significance (Dunn's post hoc multiple-comparisons test). Data were significant at $p<$ 0.05. Graphs show mean (green) + SEM (red). 
The in vivo biological activity of FGF21 is regulated by the coexpression of (a) the FGFR1c receptor and (b) the adaptor molecule KLB (a $120-\mathrm{kDa}$ protein) [19, 37]. KLB functionally links/targets circulating FGF21 to its

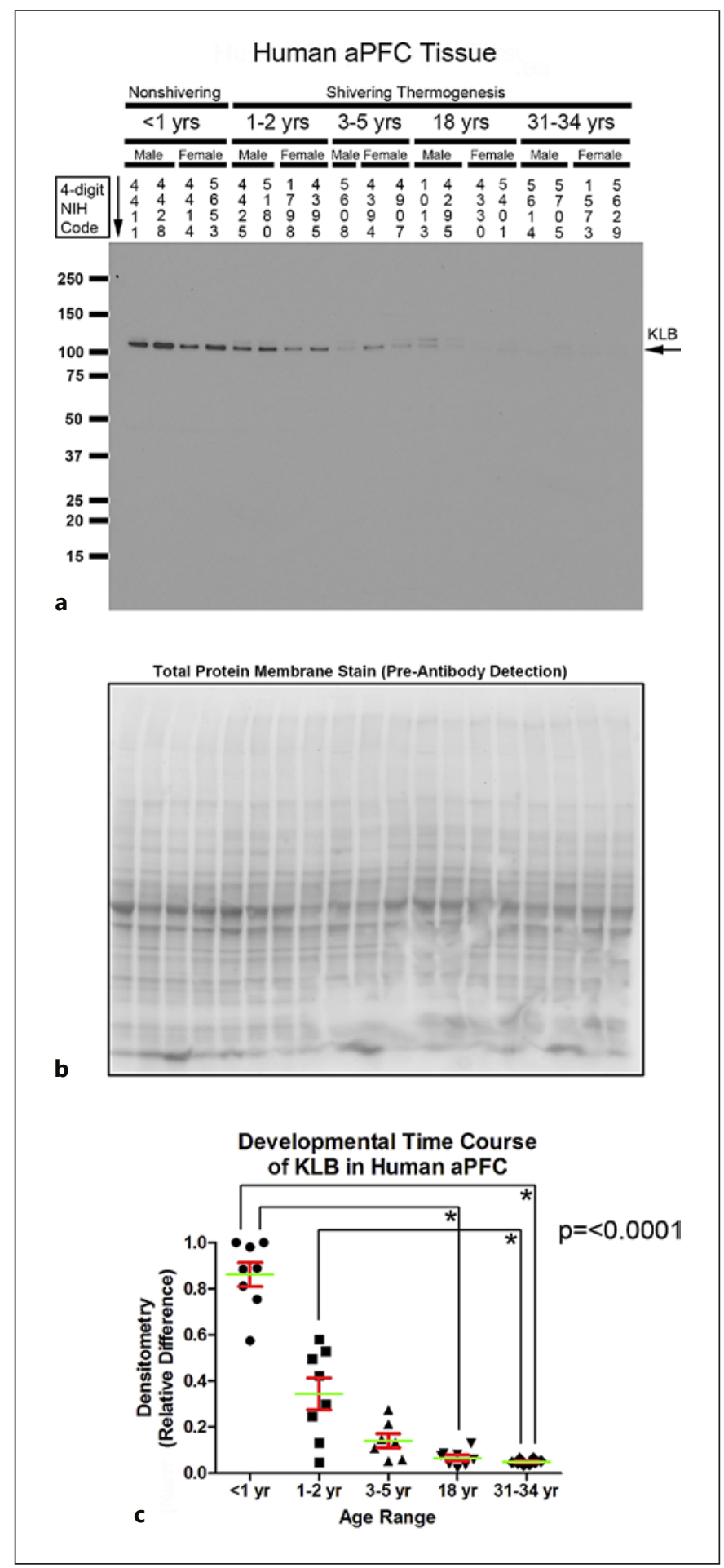

FGFR1c receptor [38]. In the adult mouse brain, FGFR1c is ubiquitous [39]. In contrast, KLB expression is restricted to just a few neuronal populations within the hypothalamus and in the hindbrain [39].

Here, we tested the hypothesis that KLB is expressed in the hippocampus and aPFC at an early developmental age in humans. Indeed, hippocampal/aPFC KLB levels were abundant in infants, moderate in toddlers, and comparatively low/absent at all other ages. The lack of KLB expression in the adult brain in regions we examined here is consistent with reports in adult mice [39]. In contrast, high levels of KLB in the hippocampus/aPFC of infants and toddlers suggest that FGF21 signaling pathways may play a larger role in human neurodevelopment than previously thought and may also be affected by total body cooling to a greater extent in infants.

\section{Study Limitations}

(1) Temperature affects CSP expression. To our knowledge, none of the subjects were administered neuroprotective cooling. However, temperature fluctuations in subjects and/or tissues could have occurred as a consequence of the wide-ranging insults leading to death prior to tissue procurement. However, our findings on CSP levels during human neurodevelopment agree with studies in rodents, in which tissues were harvested under highly controlled normothermic laboratory conditions $[10,15,27]$.

(2) Future studies must be done to examine the subcellular localization of CSP expression in the brain of human infants versus adults. In the normothermic rat brain, RBM3 colocalizes primarily with neurons and is low in glia [10]. Furthermore, RBM3 staining is almost entirely nuclear in the first postnatal week of life, shifts into the cytoplasm with increased age, and is mainly cytoplasmic in adults [10].

Fig. 8. Developmental time course of $\beta$-Klotho (KLB) expression in the human anterior prefrontal cortex (aPFC). a Representative Western blots show aPFC KLB levels in infants, toddlers, children, adolescents, and adult subjects ( $n=3$ /group in children, $n=4$ / group in all other ages). The corresponding NIH NBB deidentified subject ID is indicated above each sample. b Representative total protein stain confirms equal protein loading and membrane transfer across groups. $\mathbf{c}$ The graph shows that KLB levels are highest in the infant aPFC ( $n=8 /$ group) compared to toddlers ( $n=8 /$ group), children ( $n=7 /$ group), adolescents ( $n=8$ /group), and adults ( $\mathrm{n}=$ 8 /group). Normalized densitometry values were analyzed by Kruskal-Wallis one-way ANOVA. * significance (Dunn's post hoc multiple-comparisons test). Data were significant at $p<0.05$. Graphs show mean (green) + SEM (red). 
(3) The small sample size limited our ability to adequately test hypotheses related to individual group differences. For instance, infants visually had the highest amount of CSPs of all the age groups, but the levels varied over a relatively broad expression range. Thus, we were unable to detect a statistically significant difference by post hoc analysis for some of the comparisons even though levels appeared to be quite dramatic (i.e., RBM3 and RTN3 in infants vs. adults). Notably, the broad CSP expression range in infants may have important clinical implications. More studies are needed to elucidate if CSP expression variability in postmortem tissue in infants is due to the mechanism of insult leading to death (e.g., premature infants with SIDS), artifacts (e.g., factors involved in tissue procurement), or potentially by inherent genetic differences among patients. Germane to the latter potential mechanism of CSP expression variability, future studies are needed to test if infants with low baseline CSP levels could have increased vulnerability to a subsequent brain injury and/or represent a unique cohort of patients that might benefit most from therapeutic cooling.

(4) We cannot exclude the possibility that differences in tissue quality biased the detection of CSPs in infants versus older age groups. However, it seems unlikely, given that: (a) our results agree with highly controlled studies in rodents $[10,15,27])$, (b) a background signal de- tected by the anti-RTN3 antibody increased with advancing age, indicating that the direction of change in protein expression was not biased to a particular age group, and (c) total protein stains were included in each experiment and were unremarkable/consistent across samples.

(5) Finally, although we did not observe an effect of gender on protein expression, we cannot rule out the possibility that our study was underpowered to detect it.

\section{Acknowledgements}

We are grateful to the NIH NBB for providing human tissues used in this study. Tissues were made available upon completion and acceptance of a signed material transfer agreement between the University of Pittsburgh and the NBB. This work was supported by NIH/NINDS grants R21NS098057 and R01NS105721 to T.C.J. and the Ake N. Grenvik Chair in Critical Care Medicine to P.M.K.

\section{Disclosure Statement}

T.C.J and P.M.K. are coinventors on a pending patent on the use of FGF21 therapy in temperature-managed patients entitled: "Method to Improve Neurologic Outcomes in Temperature Managed Patients" (USPTO application No. 15/573,006).

\section{References}

1 Jacobs SE, Berg M, Hunt R, Tarnow-Mordi WO, Inder TE, Davis PG. Cooling for newborns with hypoxic ischaemic encephalopathy. Cochrane Database Syst Rev. 2013 Jan;(1):CD003311.

2 Geocadin RG, Wijdicks E, Armstrong MJ, Damian M, Mayer SA, Ornato JP, et al. Practice guideline summary: Reducing brain injury following cardiopulmonary resuscitation: Report of the Guideline Development, Dissemination, and Implementation Subcommittee of the American Academy of Neurology. Neurology. 2017 May;88(22):2141-9.

3 Arrich J, Holzer M, Havel C, Müllner M, Herkner H. Hypothermia for neuroprotection in adults after cardiopulmonary resuscitation. Cochrane Database Syst Rev. 2016 Feb; 2:CD004128.

4 Kochanek PM, Fink EL, Bell MJ, Bayir H, Clark RS. Therapeutic hypothermia: applications in pediatric cardiac arrest. J Neurotrauma. 2009 Mar;26(3):421-7.

5 Nielsen N, Wetterslev J, Cronberg T, Erlinge D, Gasche Y, Hassager C, et al; TTM Trial Investigators. Targeted temperature management at $33^{\circ} \mathrm{C}$ versus $36^{\circ} \mathrm{C}$ after cardiac arrest. N Engl J Med. 2013 Dec;369(23):2197-206.
6 Choi HA, Ko SB, Presciutti M, Fernandez L, Carpenter AM, Lesch C, et al. Prevention of shivering during therapeutic temperature modulation: the Columbia anti-shivering protocol. Neurocrit Care. 2011 Jun;14(3): 389-94.

7 Lleonart ME. A new generation of proto-oncogenes: cold-inducible RNA binding proteins. Biochim Biophys Acta. 2010 Jan; 1805(1):43-52.

8 Aherne W, Hull D. Brown adipose tissue and heat production in the newborn infant. J Pathol Bacteriol. 1966 Jan;91(1):223-34.

9 Hull D, Smales O. In: Sinclair JC, editor. Temperature regulation and energy metabolism in the newborn. New York: Grune \& Stratton; 1978.

10 Pilotte J, Cunningham BA, Edelman GM, Vanderklish PW. Developmentally regulated expression of the cold-inducible RNA-binding motif protein 3 in euthermic rat brain. Brain Res. 2009 Mar;1258:12-24.

11 Nishiyama H, Itoh K, Kaneko Y, Kishishita M, Yoshida O, Fujita J. A glycine-rich RNAbinding protein mediating cold-inducible suppression of mammalian cell growth. J Cell Biol. 1997 May;137(4):899-908.
12 Bastide A, Peretti D, Knight JR, Grosso S, Spriggs RV, Pichon X, et al. RTN3 Is a Novel Cold-Induced Protein and Mediates Neuroprotective Effects of RBM3. Curr Biol. 2017 Mar;27(5):638-50.

13 Danno S, Nishiyama H, Higashitsuji H, Yokoi $\mathrm{H}$, Xue $\mathrm{JH}$, Itoh $\mathrm{K}$, et al. Increased transcript level of RBM3, a member of the glycine-rich RNA-binding protein family, in human cells in response to cold stress. Biochem Biophys Res Commun. 1997 Jul;236(3):804-7.

14 Peretti D, Bastide A, Radford H, Verity N, Molloy C, Martin MG, et al. RBM3 mediates structural plasticity and protective effects of cooling in neurodegeneration. Nature. 2015 Feb;518(7538):236-9.

15 Chip S, Zelmer A, Ogunshola OO, FelderhoffMueser U, Nitsch C, Bührer C, et al. The RNA-binding protein RBM3 is involved in hypothermia induced neuroprotection. Neurobiol Dis. 2011 Aug;43(2):388-96.

16 Larrayoz IM, Rey-Funes M, Contartese DS, Rolón F, Sarotto A, Dorfman VB, et al. Cold Shock Proteins Are Expressed in the Retina Following Exposure to Low Temperatures. PLoS One. 2016 Aug;11(8):e0161458. 
17 Jackson TC, Manole MD, Kotermanski SE, Jackson EK, Clark RS, Kochanek PM. Cold stress protein RBM3 responds to temperature change in an ultra-sensitive manner in young neurons. Neuroscience. 2015 Oct;305:26878.

18 Hondares E, Rosell M, Gonzalez FJ, Giralt M, Iglesias R, Villarroya F. Hepatic FGF21 expression is induced at birth via PPARalpha in response to milk intake and contributes to thermogenic activation of neonatal brown fat. Cell Metab. 2010 Mar;11(3):206-12.

19 Adams AC, Cheng CC, Coskun T, Kharitonenkov A. FGF21 requires $\beta$ klotho to act in vivo. PLoS One. 2012;7(11):e49977.

20 Jackson TC, Kotermanski SE, Kochanek PM. Whole-transcriptome microarray analysis reveals regulation of Rab4 by RBM5 in neurons. Neuroscience. 2017 Oct;361:93-107.

21 Jackson TC, Bayir H, Ikonomovic MD, Janesko-Feldman K, Mi Z, Gao T, et al. Detection of PHLPP $1 \alpha / \beta$ in human and mouse brain by different anti-PHLPP1 antibodies. Sci Rep. 2015 Apr;5(1):9377.

22 Jackson TC, Du L, Janesko-Feldman K, Vagni VA, Dezfulian C, Poloyac SM, et al. The nuclear splicing factor RNA binding motif 5 promotes caspase activation in human neuronal cells, and increases after traumatic brain injury in mice. J Cereb Blood Flow Metab. 2015 Mar;35(4):655-66.

23 Jackson TC, Kotermanski SE, Jackson EK, Kochanek PM. BrainPhys ${ }^{\circledR}$ increases neurofilament levels in CNS cultures, and facilitates investigation of axonal damage after a mechanical stretch-injury in vitro. Exp Neurol. 2018 Feb;300:232-46.
24 Collins MA, An J, Peller D, Bowser R. Total protein is an effective loading control for cerebrospinal fluid western blots. J Neurosci Methods. 2015 Aug;251:72-82.

25 Moritz CP. Tubulin or Not Tubulin: Heading Toward Total Protein Staining as Loading Control in Western Blots. Proteomics. 2017 Oct;17(20):17.

26 Bond JF, Farmer SR. Regulation of tubulin and actin mRNA production in rat brain: expression of a new beta-tubulin mRNA with development. Mol Cell Biol. 1983 Aug;3(8): 1333-42.

27 Di Scala F, Dupuis L, Gaiddon C, De Tapia M, Jokic N, Gonzalez de Aguilar JL, et al. Tissue specificity and regulation of the N-terminal diversity of reticulon 3. Biochem J. 2005 Jan; 385(Pt 1):125-34.

28 Silasi G, Colbourne F. Therapeutic hypothermia influences cell genesis and survival in the rat hippocampus following global ischemia. J Cereb Blood Flow Metab. 2011 Aug;31(8): 1725-35.

29 Shaw P, Greenstein D, Lerch J, Clasen L, Lenroot R, Gogtay N, et al. Intellectual ability and cortical development in children and adolescents. Nature. 2006 Mar;440(7084):676-9.

30 Casey BJ, Giedd JN, Thomas KM. Structural and functional brain development and its relation to cognitive development. Biol Psychol. 2000 Oct;54(1-3):241-57.

31 Sakurai T, Kashida H, Watanabe T, Hagiwara $\mathrm{S}$, Mizushima T, Iijima $\mathrm{H}$, et al. Stress response protein cirp links inflammation and tumorigenesis in colitis-associated cancer. Cancer Res. 2014 Nov;74(21):6119-28.

32 Qiang X, Yang WL, Wu R, Zhou M, Jacob A, Dong W, et al. Cold-inducible RNA-binding protein (CIRP) triggers inflammatory responses in hemorrhagic shock and sepsis. Nat Med. 2013 Nov;19(11):1489-95.
33 Zhou M, Yang WL, Ji Y, Qiang X, Wang P. Cold-inducible RNA-binding protein mediates neuroinflammation in cerebral ischemia. Biochim Biophys Acta. 2014 Jul;1840(7): 2253-61.

34 Bal NC, Maurya SK, Sopariwala DH, Sahoo SK, Gupta SC, Shaikh SA, et al. Sarcolipin is a newly identified regulator of muscle-based thermogenesis in mammals. Nat Med. 2012 Oct;18(10):1575-9.

35 Sánchez-Infantes D, Gallego-Escuredo JM, Díaz M, Aragonés G, Sebastiani G, LópezBermejo A, et al. Circulating FGF19 and FGF21 surge in early infancy from infra- to supra-adult concentrations. Int J Obes. 2015 May;39(5):742-6.

36 Keipert S, Kutschke M, Ost M, Schwarzmayr T, van Schothorst EM, Lamp D, et al. LongTerm Cold Adaptation Does Not Require FGF21 or UCP1. Cell Metab. 2017 Aug;26(2): 437-446.e5.

37 Kurosu H, Choi M, Ogawa Y, Dickson AS, Goetz R, Eliseenkova AV, et al. Tissue-specific expression of $\beta$-klotho and fibroblast growth factor (FGF) receptor isoforms determines metabolic activity of FGF19 and FGF21. J Biol Chem. 2007 Sep;282(37): 26687-95.

38 Lee S, Choi J, Mohanty J, Sousa LP, Tome F, Pardon E, et al. Structures of $\beta$-klotho reveal a 'zip code'-like mechanism for endocrine FGF signalling. Nature. 2018 Jan;553(7689): 501-5.

39 Bookout AL, de Groot MH, Owen BM, Lee S, Gautron L, Lawrence HL, et al. FGF21 regulates metabolism and circadian behavior by acting on the nervous system. Nat Med. 2013 Sep;19(9):1147-52. 